\title{
THE HEIDELBERG PNEUMATIC ARM PROSTHESIS
}

\author{
Ernst Marquardt, Heidelberg, West Germany \\ From the Orthopaedic Clinic of Heidelberg University \\ (Director, Professor Dr K. Lindemann)
}

The equipping with active arm prostheses of patients with high level bilateral upper arm amputations and of patients with bilateral shoulder disarticulations still presents us with great difficulties. Control of the necessary active joint movements and their locking with a strength suitable for everyday and occupational use becomes more problematical with the increasing height of the amputation level.

Realising these difficulties, S. Weil, at that time director of this clinic, began in 1948 to experiment with the carbon dioxide prosthesis invented by Häfner, especially for high level arm amputations and shoulder disarticulations. Since 1955 I have had the opportunity, in the service headed by Professor Lindemann, to continue with the further development of the pneumatic prosthesis, especially in cases difficult to fit and in children. I worked first in collaboration with Dr O. Häfner and from 1958 onwards with Drs A. Emmerich and H. Kramer.

In the Heidelberg pneumatic prosthesis compressed carbon dioxide is used as the source of power. It thus has all the disadvantages inherent in the use of an external power supply, such as the need to replenish the power supply and to provide care and servicing. With all our enthusiasm about modern technical achievements we must clearly recognise and bear in mind the disadvantages. The state of development of conventional arm prostheses with cable control is so far advanced that we must make specially high demands on externally powered prostheses. In the construction of arm prostheses an external source of power will therefore only succeed if it really offers the amputee some advantages, namely 1) in the endurance of activity; 2) in the power obtainable for necessary movements and lockings; 3 ) in the number of the movements required; and 4) in the control.

The knowledge gained in the construction of arm prostheses generally must also be considered in externally powered prostheses, because the total performance of the prosthesis depends on the material used and its technique of manufacture. It also depends on the nature of the prehensile organ and joints, on the type of prosthesis control and especially on the technique of fitting and through that on the quality of the union between body and prosthesis. An arm prosthesis will not be very efficient if, in spite of an ingenious control mechanism, the hand substitute does not stand up to everyday life and occupational requirements, but is more of an ornament. The efficiency of a prosthesis also suffers, especially in cases of high amputations and shoulder disarticulations, if the pressing necessity of pronation and supination is forgone, if the essential fixations and locks are not provided and also if the amputee does not get a sensory " report " about the strength of grip. Mistakes like a faulty position of the control mechanism and overaction of voluntarily moved joints can also cause the fitting of a prosthesis to end in failure. During our development work on the pneumatic prostheses we have not been spared these mistakes. We consider it our duty to make this generally known so that the same mistakes may be avoided by other research teams from the start. It is understandable if the inventor of a new type of prosthesis believes that his prosthesis is the best and most advantageous for all arm amputees. All the same it is essential that the indications for the use of the particular types of prosthesis be clearly defined and recognised. It must also be stressed that not every external source of power has advantages for the arm amputee. In $1955 \mathrm{Dr}$ Häfner and I formulated the requirements of an external source of 
power in the construction of arm prostheses, as follows: 1) It must give all joint movements energy as close as possible to that of the natural power of an arm. 2) It must be capable of fine graduations and allow smooth and flexible transmission. 3) For small movements the control should require little power only. 4) No power should be lost through continuous loading of joints. 5) The material used to provide power should not be expensive, heavy or bulky; it should be easily replenished and should last for at least one day without refilling when the prosthesis is in full time use.

We found that carbon dioxide possessed these properties. Its use as an external power source demands, however, that measures be taken to protect the patient and his surroundings. This was particularly stressed by Kiessling (1961). Kiessling concluded that carbon dioxide is one of the most important external sources of power for prostheses and splints provided the usual safety regulations are observed.

Weil described the principle of the pneumatic prosthesis in 1954: " The patient carries on his body or within the prosthesis a small aluminium bottle filled with liquid carbon dioxide. The carbon dioxide passes through a reducing valve into a rubber tube and rubber bellows which stretch on filling and cause the desired movement. This extremely simple system is operated by the disabled person by regulating valves; that is, he opens or closes the passage for the carbon dioxide, for instance by a pressing movement, by the shoulder, by traction valve or by stump rotation and, above all, by alternating contraction and expansion of the musculature of the stump (the "muscle valves ')." This principle has not been changed since then though essential development work has further increased the safety and efficiency of the pneumatic prosthesis. Thus we use today steel containers in place of aluminium, and we use plastic instead of rubber for the tubes. Special cylinders with pistons and diaphragms have been added to the bellows, and not least the artificial muscle devised by McKibben that is so important for apparatus to aid the paralysed. New developments include prostheses for small children, pneumatic hooks and pneumatically lockable shoulder joints. Control valves and most of the existing pneumatic joints were developed further. Since December 1962 the firm of Otto Bock, Duderstadt, has been associated with this development work. The development workshops of the orthopaedic clinics in Münster and Hanover also take part in the further development of the pneumatic system.

The advances in the technique of fitting the prostheses and especially of the controls to the body have led to a remarkable increase in efficiency in the use of pneumatic prostheses. A lecture by $\mathrm{Mr}$ Capener in 1959 about the problem of right and left handedness was of real help to me in the fitting of the prosthesis and control mechanism. In this connection I refer to my (1964) article.

Experience gained in fitting more than 350 adults, juveniles and children, amongst them sixty small children, with pneumatic prostheses and in detailed follow-up examinations has given us knowledge about the indications. The table of indications for fitting the pneumatic arm prosthesis (Marquardt 1959) is in our opinion still fully valid. Today, severe malformations of limbs and high level bilateral arm amputations provide the most important indication for the fitting of pneumatic prostheses. The main reasons for this are 1) the powerful grip of the hand prosthesis (or of the pneumatic hook), 2) the voluntary pronation and supination which depend on the movement of other joints, and 3) the simple control mechanism with the possibility of a certain amount of sensory report on the gripping power.

\section{CASE REPORT}

Case 1-A twenty-two-year-old man lost both upper limbs including the scapulae at the age of eighteen through a conveyor belt accident. He thus presents an extremely rare case of bilateral forequarter amputation (Fig. 1). There was severe scarring with partial loss of thoracic musculature and considerable limitation of movement of the spine, especially in the 


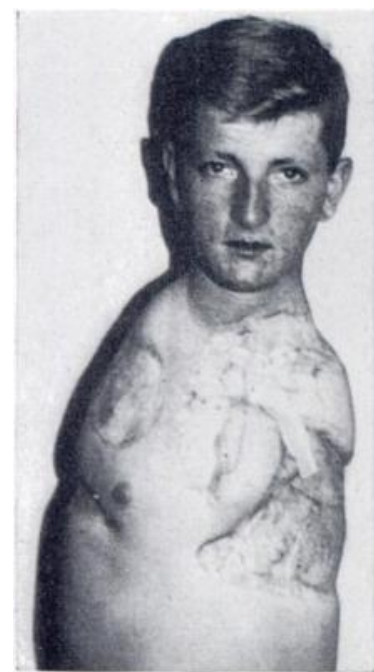

FIG. 1

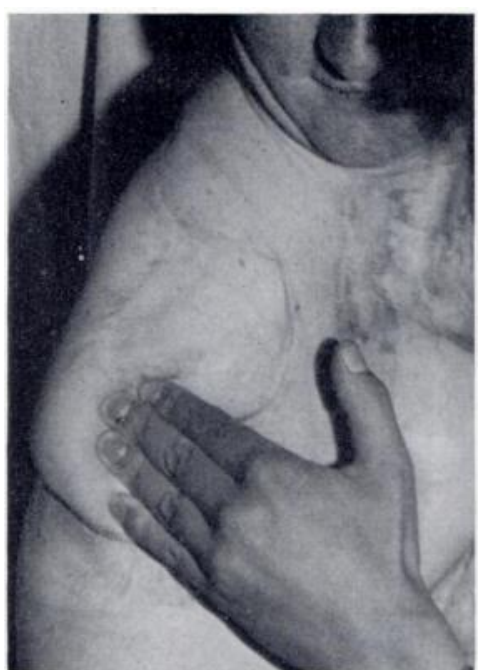

Fig. 2

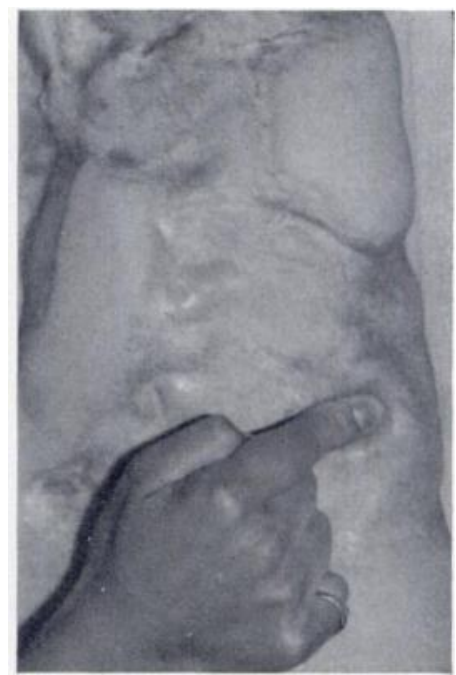

Fig. 3

Case 1. Figure 1-Man aged 22 years. Bilateral shoulder girdle amputation (interscapulothoracic amputation) by avulsion of both arms by a conveyor belt. Figures 2 and 3-The patient is asked to contract the right pectoralis major muscle (Fig. 2) and the left serratus anterior muscle (Fig. 3) against the finger pressure of the physiotherapist. With graduated increase of pressure a good result can be achieved.

thoracic region. When the patient was admitted to hospital in February $1964 \mathrm{I}$ had no idea how a prosthesis could be controlled. The right pectoralis major muscle and the partially preserved left serratus anterior muscle were acting. But it could not be predicted whether control of the prosthesis could ever be achieved with the help of these muscles. We started by training the two muscles to contract against the graduated resistance of the physiotherapist's fingers (Figs. 2 and 3), and by physical training including swimming. Because at first the fitting of a prosthesis was not possible, foot exercises in our occupational therapy department were added. We were surprised that a twenty-two-year-old man was still capable of achieving such great dexterity with both feet (Fig. 4). This dexterity is also useful to the patient in everyday life, though he could not manage to learn to eat, drink and write with his feet. When the muscle belly of the pectoralis major and the remainder of the serratus muscle had achieved a lift of approximately one centimetre on contraction we started with the fitting of a prosthesis (Fig. 5). Over the right pectoralis muscle we fitted a double pressure ball valve to control the closing and opening of the hand.

Control of grip-The muscle power needed to press down the ball rises in the proportion to the power of the hand grip. The thumb of the pneumatic hand prosthesis begins to close when the ball effecting the closure is loaded by approximately 250 grammes. The tight closure of the hand against a thumb end pressure of approximately 10 kilograms is only reached when the ball is loaded with approximately 450 grammes. In this way a certain amount of " report"

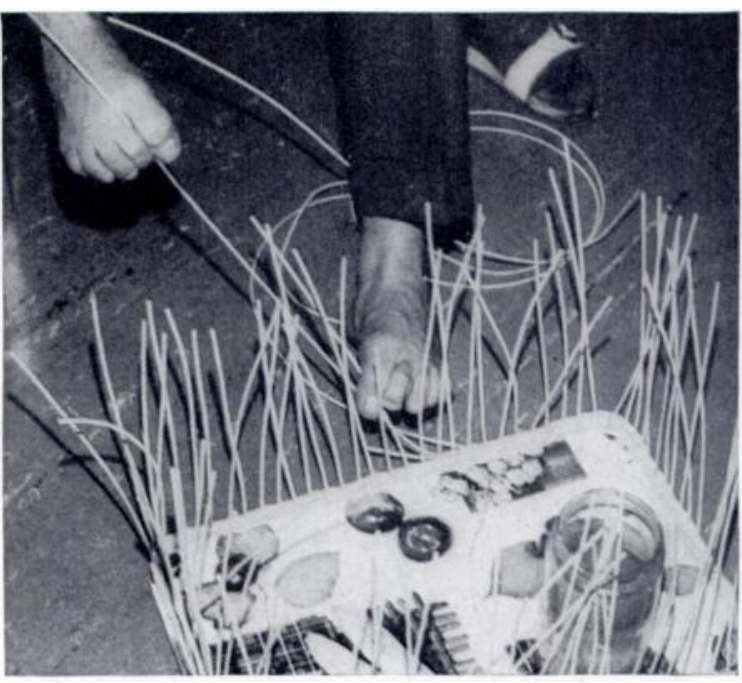

FIG. 4

Case 1-Training of feet to increase the patient's ability to help himself. 

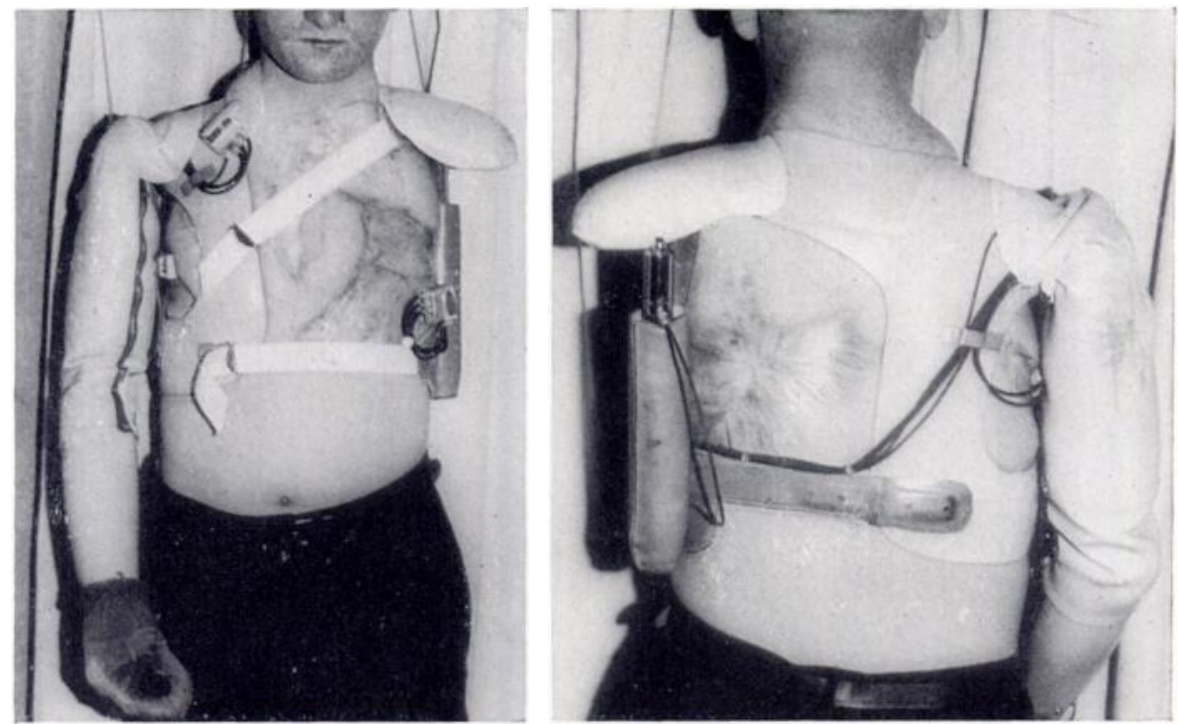

Fig. 5

Case 1-Fitting of a right-sided pneumatic prosthesis. The gripping function is controlled by the right pectoralis major muscle through a double pressure valve. Rotation of the hand (170 degrees) with automatic locking is controlled by the left serratus anterior muscle through a quadruple pressure valve, while elbow movements with voluntary locking are effected by a quadruple chin pressure valve. The remaining joints (bi-axial shoulder joint; upper arm rotation joint) are passively adjustable locking friction joints. The carbon dioxide container is fitted to the left side to balance the weight.
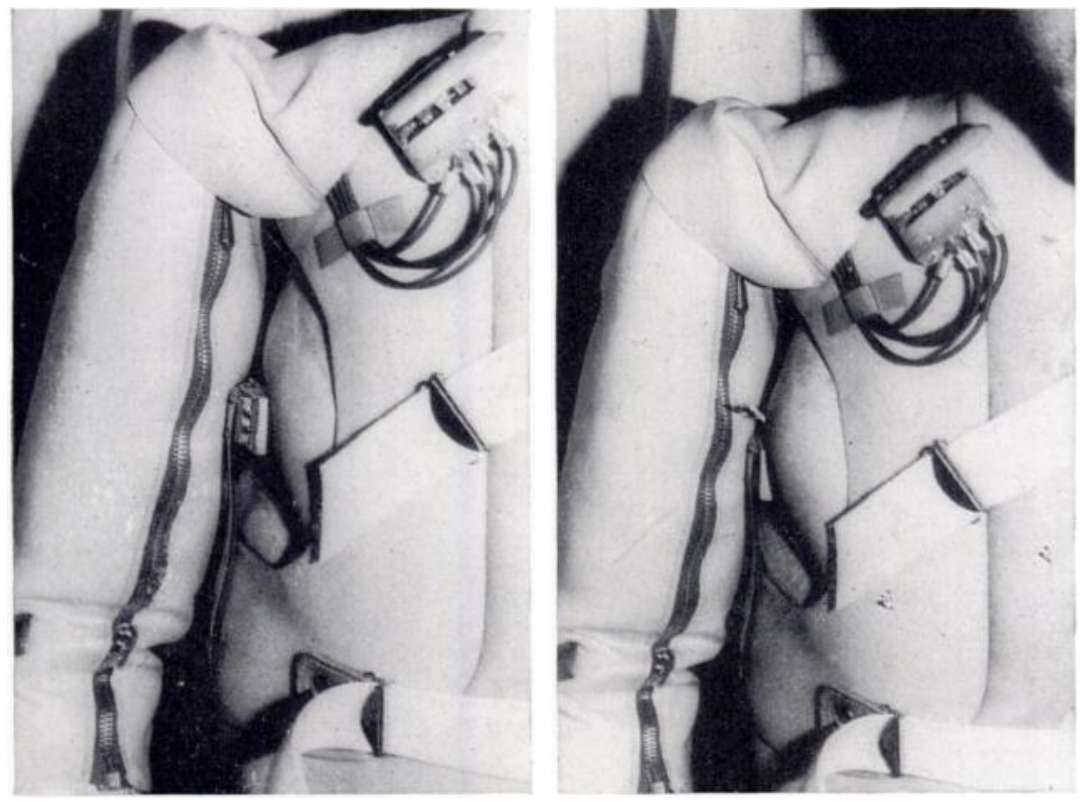

Fig. 6

Case 1-The application of the double pressure valve to the right pectoralis major muscle: relaxed, and contracted (right). In the relaxed state (left) the control balls of the valve are easily visible. The chin pressure valve is also well shown in this figure. 
on the gripping power is possible. Figure 6 shows the fitted control valve with relaxed and with contracted pectoralis major muscle. The first valve point effects the closure, the second the opening of the hand; thus the patient requires a lesser amount of muscle contraction for achieving closure of the hand than for reaching the second valve point. This is a ball valve of similar construction, allowing the escape of carbon dioxide from the bellows or cylinder and thereby effecting the opening of the hand. This arrangement is necessary for two reasons. Firstly, in the initial phase of contraction a more delicately graduated and so more accurate control of the hand or joint function is possible than with the less easily controllable maximum muscle contraction. And secondly, with a reversal of this sequence and with an accidental slight touch of the opening point, objects held in the hand could easily be dropped.

Our patients have quickly got used to our choice of arrangement. And we have not encountered any difficulties with the use of the twofold muscle pressure valve in forearm stumps or as biceps valve in upper arm stumps.
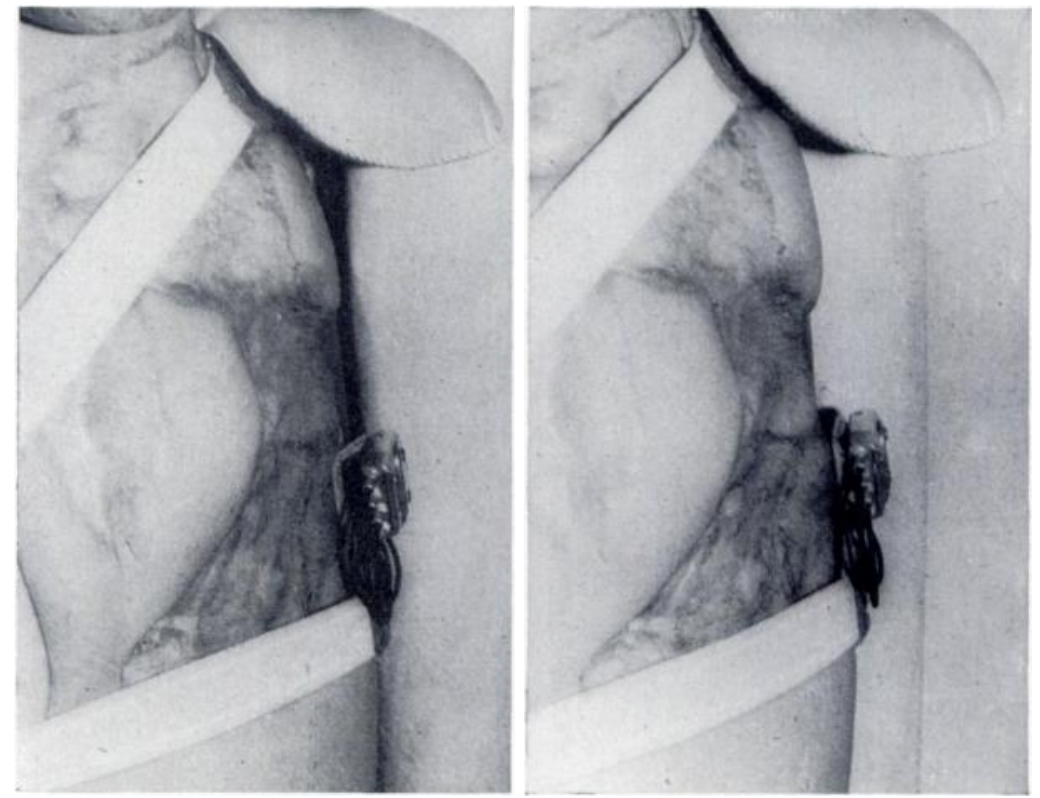

FIG. ?

Case 1-The fitting of the quadruple pressure valve to the left serratus anterior muscle to control hand rotation is shown: with relaxed and with contracted muscle (right).

Control of rotation-Figure 7 shows the fitting of a fourfold pressure valve to the relaxed left serratus anterior muscle. This fourfold pressure valve controls the rotation of the opposite hand through variation in the state of muscular contraction. One must use a fourfold valve because in the adult automatic locking at any given position of rotation is essential. Only thus, with proper fitting and with locking at all joints, will a pencil or pen, held in the hand, follow the thoracic movements without play. Any wobbling caused by faulty fitting of the plastic suction cup to the thorax or by springy or swinging out joints would jeopardise the practical use of the prosthesis. In contrast to the chin pressure valve destined for the right pneumatic elbow joint (Fig. 6) the fourfold pressure valve is constructed in such a way that with relaxation of the muscle a cam locks the movement, so that any position taken up remains locked automatically until the valve is used again.

Because of the severity of the injury we could not achieve muscular control of the elbow joint. We therefore used chin control which already had proved itself in small children. This control produces elbow flexion by strong flexion of the head, extension of the elbow by

vol. 47 B, No. 3, AUGUST 1965 
a slight retraction of the chin, a free swing by quick return to the neutral position and locking of the elbow joint by a slow return to this position. With this type of control one uses not only the direction of the control movement but also its speed in activating the valve. Devious as chin control may appear at first it is perfectly reasonable. Flexion of the head and raising of the hand towards the mouth, retraction of the head and the simultaneous withdrawal of the

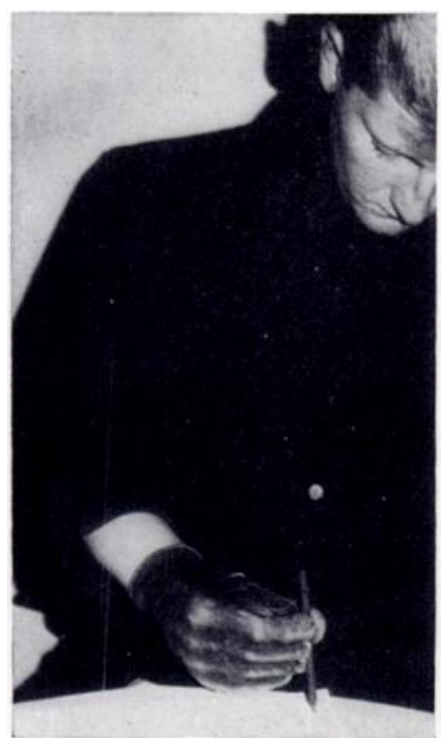

Fig. 8

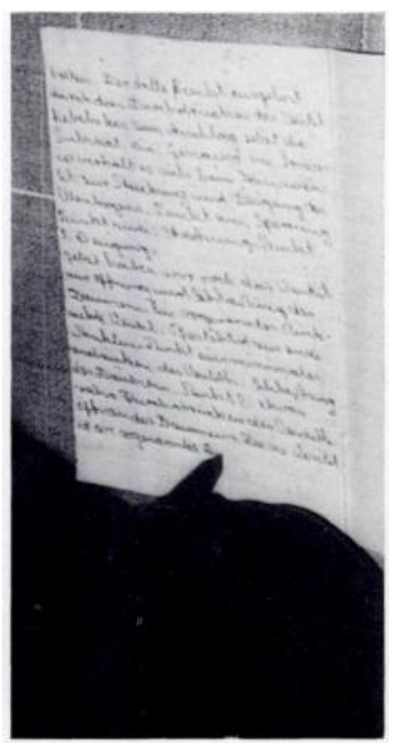

Fig. 9

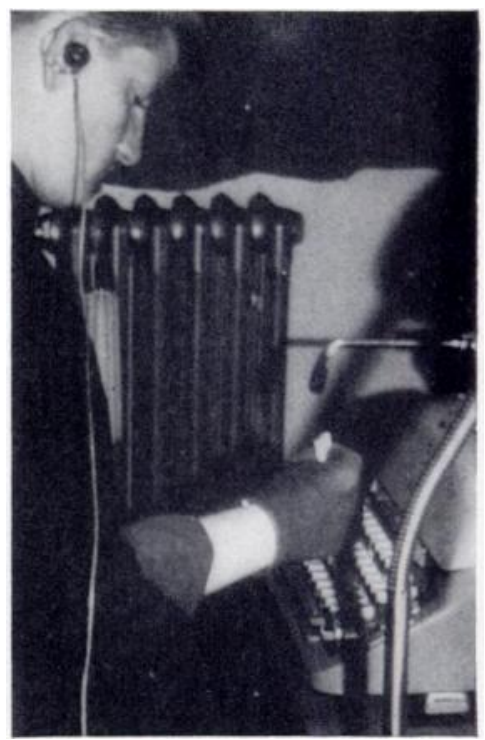

Fig. 10

Writing with the help of a prosthesis with ballpoint pen (Figs. 8 and 9) and with a typewriter (Fig. 10).

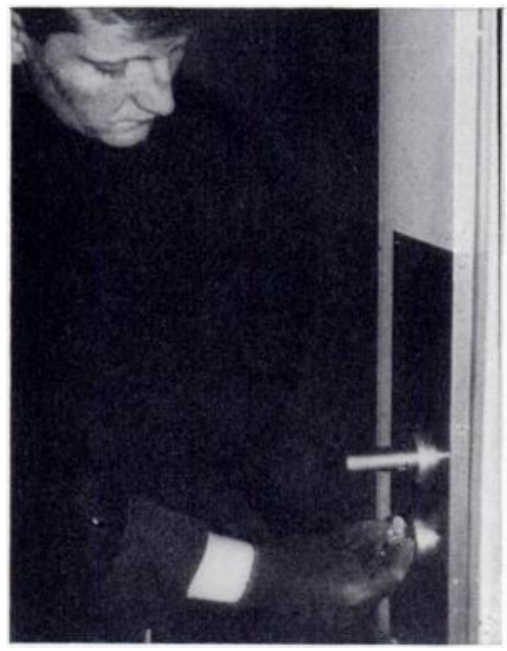

Fig. 11

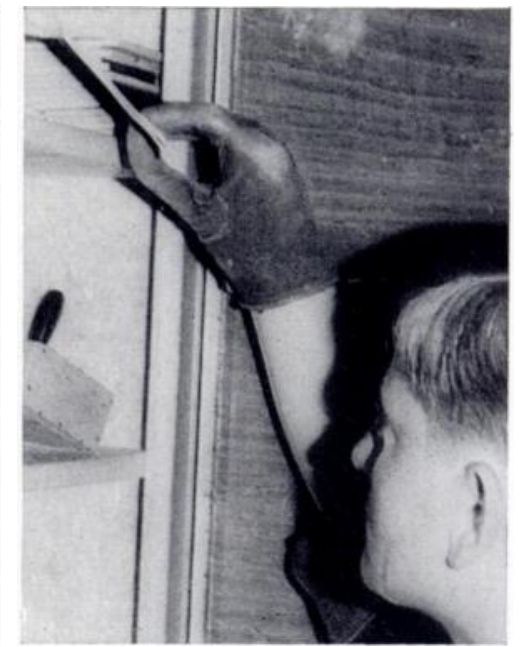

FIG. 12

Figure 11-Active pronation and supination are two of the most important movements in everyday life. Figure 12-Through muscular control independent of a shoulder strap objects can be grasped above the horizontal level if the shoulder joint is suitably adjusted.

hand from the mouth, are in every way physiologically coordinated movements. Furthermore, with chin control the adjustment of the elbow joint remains visually well controllable.

All three control valves can be used voluntarily and independently and allow coordinated movements of hand rotation and gripping. As already mentioned, the control is so sensitive that the patient can accurately regulate the adjustment of the grip with this valve. 
In addition to the active joint movements a passively adjustable upper arm rotation device is fitted above the elbow joint as well as a passively adjustable lockable bi-axial shoulder joint. In cases like this, especially if there are sensitive scars in the shoulder area, we have lately used a pneumatic voluntarily lockable shoulder joint fitted with serrated discs. Both serrated discs are pressed together by a pressure of $\mathbf{4 0}$ kilograms while for unlocking they are separated by plate springs, thus allowing the arm to swing freely. In this way the scars are less subjected to pushing and chafing movements. Furthermore, if elbow flexion is strong, unlocking of the shoulder joint allows gripped articles easily to be brought up in an axial direction-as for instance trousers after use of the toilet.

On account of the extensive severe scar formation a bilateral fitting was not possible. In order to balance the weight the carbon dioxide container is carried on the left side of the body.

With his pneumatic prosthesis this severely injured man learned in a relatively short time to eat, drink and write with pencil, ballpoint pen and typewriter (Figs. 8 to 10), to urinate without help and to perform many functions of everyday life by himself (Figs. 11 and 12). He uses his well trained feet to insert paper into the typewriter.
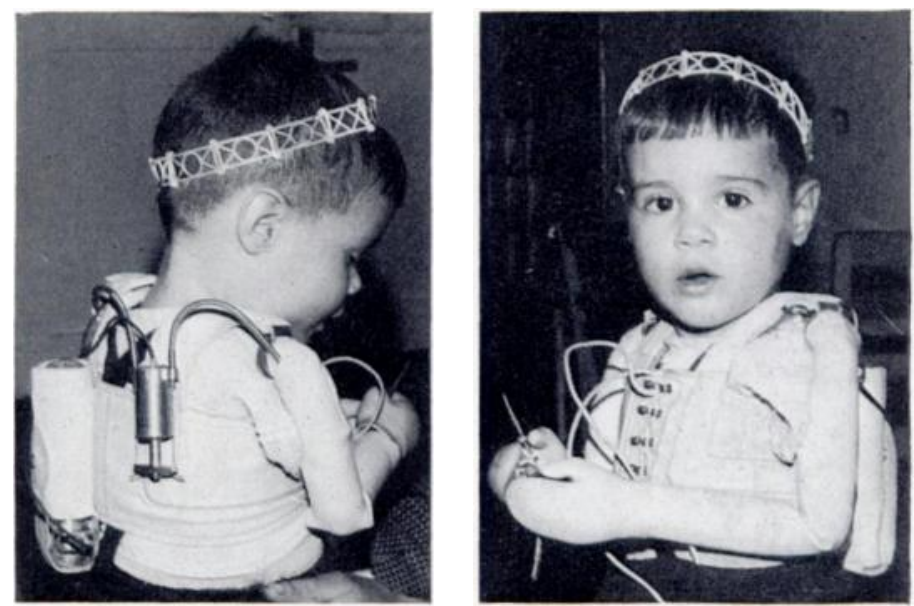

Fig. 13

Two-year-old boy with bilateral amelia, fitted with pneumatic paw hand prostheses. The carbon dioxide container and the working cylinder with valves are carried on the back. Two Bowden cables lead from the piston rod to the arm rotating joints. By means of the tapping-key valve fitted to the boy's shoulder (with a stirrup encircling the shoulder) a coupled medial rotation and apposition of the paw hands or a coupled lateral rotation and separation of the paws is effected. Movement can also be controlled by twin valves or in cases of phocomelia by tapping-key valves worked by the small finger (compare Fig. 15).

Figure 9 shows the patient's handwriting with prosthesis after six months' training. This shows that the character of the writing with the prosthetic hand becomes similar to that before the accident and indicates that, as Lindemann has pointed out, the character of the handwriting depends more on the central nervous system than on the periphery.

\section{SPECIAL PROBLEMS IN CHILDREN}

In children the type of prosthesis and the age at which it is fitted depend on the physical and mental state of development and on the type of malformation. In bilateral amelia and also in bilateral complete phocomelia we start in the first place with the fitting of a pneumatic paw hand prosthesis (Fig. 13). We do this as soon as the child has learnt to grip and touch with his feet and is able to sit up properly. In patients with well developed shoulders a twin traction valve system operating through a cylinder and pistons and Bowden cables controls

vol. 47 B, No. 3, AUguSt 1965 
the coupled medial rotation of both arms by a forward movement of the shoulders. This, with elbows bent at right angles, allows a gripping movement of the two simple baby paw hands. When the shoulders are moved backwards the other half of the twin valve acts and produces coupled lateral rotation and thereby opening of the grip. In cases with hypoplastic shoulders a " tapping key" valve is fitted with an elongated stirrup. This stirrup is fitted to one shoulder. Forward movement of the shoulder causes coupled medial rotation and backward movement causes coupled lateral rotation by the appropriate touching of the valve points.

In cases of phocomelia we use the small hand to control the prosthesis. Paw hand prostheses can be used for children of one to two years. The child so fitted gets accustomed
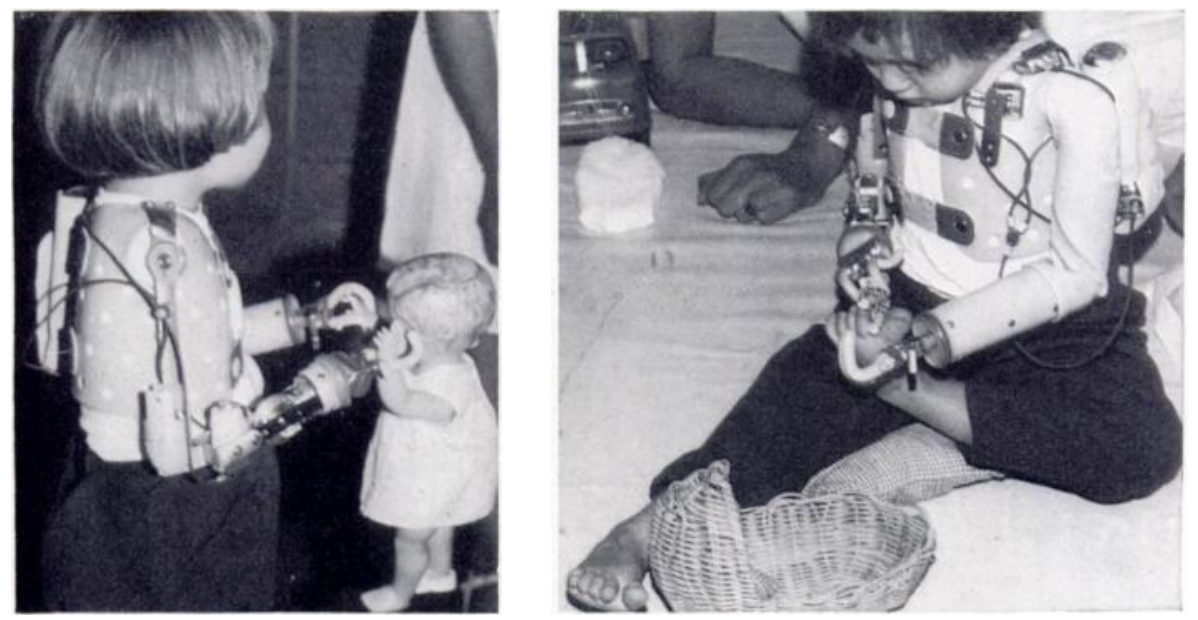

FIG. 14

Two-year-old boy with bilateral one-finger phocomelia. By means of a tapping-key valve the right finger controls the right pneumatic hook and the left finger the left hook. At the front of the plastic suction cup a twin traction valve is fitted, which engages on retraction of the shoulders and causes a coupled lateral rotation of both arms; a corresponding valve is fitted at the back which activates coupled medial rotation when the shoulders are brought forward. The boy now is 3 years old: recently a right-sided pneumatic hand rotation has been fitted. This and the right hook are controlled by the right finger (the left finger was too weak). Ability active to pronate and supinate improves the performance considerably.
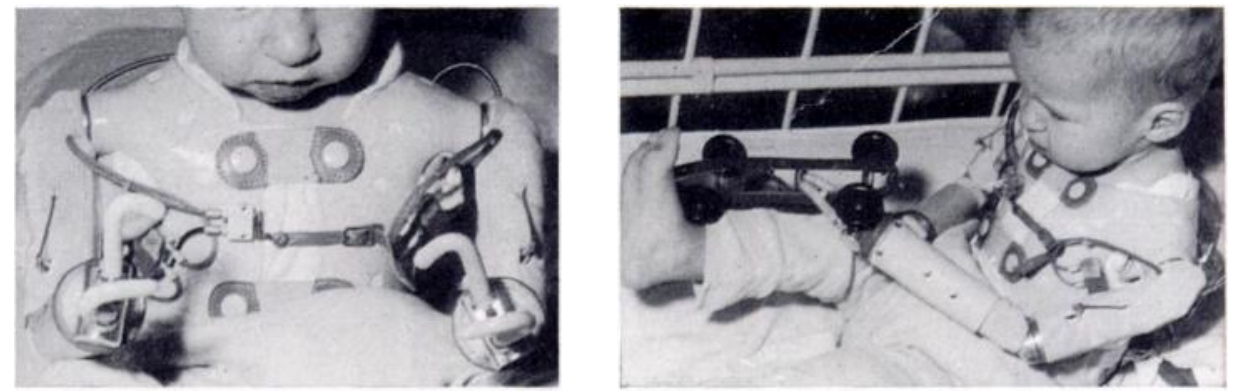

FIG. 15

Fitting of active pneumatic hooks to both sides in a case of bilateral one-finger phocomelia.

early to the use of artificial arms, includes them into his body pattern as his own arms and uses them for simple functions normally performed by children, such as gripping and holding of dolls, building blocks and balls and pushing of prams. At two to three years we have to change the type of prosthesis completely. We then give them passive upper arm rotation joints, passive elbow joints, an active hand rotating joint and an active hook on the dominant side in addition to the passive shoulder joints already present. In Figure 14 such a fitting on a child of two and three-quarter years with bilateral amelia can be seen. Control of the 
gripping function of the right-sided hook is effected by forward movement of the left shoulder, and rotation of the right pneumatic hand joint is controlled by elevation of the left shoulder. If the child moves its left shoulder forward and upward it can, depending on the way it executes this movement, perform coordinated grip and rotation movements with the right hand substitute. In cases of bilateral one-finger phocomelia we can connect an active pneumatic hook on both sides immediately (Fig. 15).

At the age of four or five years active pneumatic elbow joints are added. They are best controlled by chin pressure valves.

Children with bilateral phocomelic ectromelia-by this we understand the so-called " connecting piece" phocomelia-mostly need a prosthesis only on the functionally worse side. Provided there is an adequately long connecting piece the hands usually enable the child to eat, drink and play independently, if it is functionally well trained. Simple eating and writing aids are often more efficient in such cases than complicated prostheses. Children with short connecting pieces, however, are usually unable to reach one hand with the other for play. We then fit the functionally worse side with a so-called ectromelia type of pneumatic

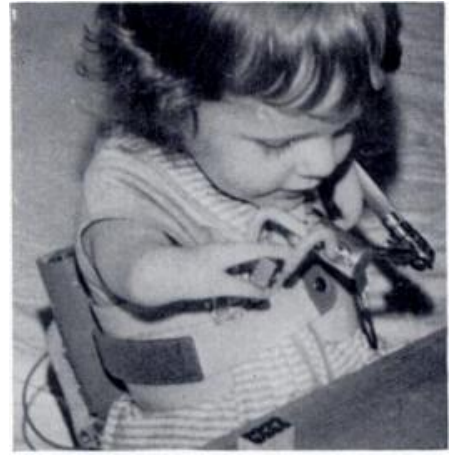

FIG. 16

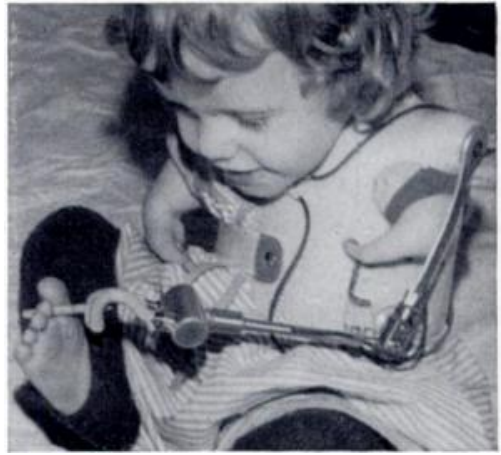

Fig. 17

Three-year-old girl with phocomelic ectromelia on the right and phocomelia on the left. The right hand enables the child to eat without help. " Hand in hand " play was only possible after fitting a prosthesis. The left extremely weak finger operates the powerful grip of the pneumatic hook by a special valve construction. Figure 16-Combined play of the right hand and the left prosthesis. Figure 17Combined play of the right foot with the left prosthesis. In this connection the importance of foot exercises in all cases of amelia, phocomelia and phocomelic ectromelia of the upper extremities must be stressed. To help themselves these children will largely depend on the use of their feet as the best substitute for their hands, in spite of the best prostheses.

prosthesis, which, as an innovation, does not include the phocomelic hand in a shaft, but uses it for manipulation of the prosthesis. In this way the child can also use the hand directly if the clothing has been altered suitably. The most important new development in cases of phocomelia and phocomelic ectromelia is that the prosthetic hand is controlled by the malformed hand. The passively controlled shoulder joint of this prosthesis is fitted eccentrically. We developed it at first as a passive locking joint but have now developed a pneumatically lockable shoulder joint for small children which can be worked by a twofold pressure valve. The disc or cone-shaped brake allows free swinging of the arm when unlocked, and with suitable use of the valve an increasing braking action to complete locking.

In cases of one-finger phocomelia, or at times phocomelic hands, the finger may sometimes be so weak that our usual tapping-key valves cannot be operated. We then use a rotary valve with a long lever and, if necessary, with a ring attached for the small finger, or a modified long-levered twin-traction valve system. Children who can only perform a weak pulling movement but cannot exert any pressure with their small fingers attached to the shoulder, clasp or grip the lever and pull it up to the shoulder (Figs. 16 and 17). The valve illustrated 
causes the hook to begin closing at a pull of 28 grammes while a pull of 240 grammes enables the small child's pneumatic hook to be firmly closed against a final load of 1,250 grammes. In this case we arranged the opening of the hook by chin pressure with a specially modified tapping-key valve. After successful strengthening of the small finger one can change over to a conventional tapping-key valve or to a rotary valve equipped with a touch lever or even a twofold pressure valve.

So far we have equipped sixty children with pneumatic prostheses. We have found that the use of the control valves is in no way too complicated for these small children, provided proper training is given. In the occupational therapy department the children learn to use the prosthesis during play and accept the pneumatic hooks as their hands more quickly and more easily than adults. Success depends, apart from our effort, on the cooperation of the parents. The training of parents thus occupies a big space in our prosthesis programme for children. Failures with children's prostheses are mostly caused by the wrong attitude of the parents. Besides the institutional fitting of prostheses and the training, which includes that of parents, we regularly conduct consulting sessions with the whole dysmelia team taking part. This team consists of doctor, psychologist, occupational therapist, physiotherapist and foreman of the workshop; it also includes the social worker, nursery school teacher and nurses. In the centre of the team, however, is the dysmelic child and its parents. During the consulting session all physical and psychological problems are discussed; the programme is being extended and built up. At certain intervals, which depend on the peculiarities of the individual case, renewed institutional treatment becomes necessary, especially when new equipment has been fitted.

Training in the use of the prosthesis naturally occupies an important part in the care and rehabilitation of the adult patient. In adults, however, success depends in a much greater degree on the determination of the patient than in children. In this article I have only mentioned the main indications for the use of the pneumatic prosthesis. I would like to mention, however, our experiments with bilateral forearm amputees and forearm and opposite upper arm amputees, and further, with cases of unilateral arm amputations and limb malformations. Here a pneumatic prosthesis is advantageous in all those cases where, provided a technical understanding is present, control by stump or by grip or rotation of a dysmelic hand is possible.

In forearm amputees we have for years favoured the open splint construction, which, in contrast to the closed open splint construction, allows the skin sensitivity of the stump to be used. Further experiments are being conducted to combine the pneumatic with the electrical system. We are engaged in the project of working the pneumatic valves with the help of electromagnets in special cases. This is in preparation for the eventual electronic control of pneumatic prostheses about which more will be reported in due course.

\section{REFERENCES}

Capener, N. (1960): Prosihetics International. Proceedings of the Second International Prosthetics Course, Copenhagen, 133.

Kiessling, E. A. (1961): Publication 874 of the National Academy of Sciences, Washington, 1961.

Marquardt, E. (1959): Prostheses, Braces and Technical Aids, No. 6. Copenhagen.

MARQuardT, E. (1964): Artificial Limbs. In Book of the Year 1964 of the Encyclopaedia Brittanica.

MARQUARDT, E., and HäFNER, O. (1956): Technische Bewährung und praktische Anwendung der Heidelberger pneumatischen Prosthese. Archiv für Orthopädische und Unfall-Chirurgie, 48, 115.

WeIL, S. (1954): Arm und Armprothesen. Schweizerische Medizinische Wochenschrift, 84, 1018. 